\title{
Superconducting Coherence Length and Magnetic Penetration Depth of a $p$-wave Holographic Superconductor
}

\author{
Hua-Bi Zeng ${ }^{1}$, Zhe-Yong Fan ${ }^{1}$, and Hong-Shi Zong ${ }^{1,2}$ \\ 1 Department of Physics, Nanjing University, Nanjing 210093, China and \\ 2 Joint Center for Particle, Nuclear Physics and Cosmology, Nanjing 210093, China
}

\begin{abstract}
A classical $S U(2)$ Einstein-Yang-Mills theory in 3+1 dimensional anti-de Sitter spacetime is believed to be dual to a $p$-wave superconductor in $2+1$ dimensional flat spacetime. In order to calculate the superconducting coherence length $\xi$ of the holographic superconductor near the superconducting phase transition point, we study the perturbation of the gravity theory analytically. The superconductiong coherence length $\xi$ is found to be proportional to $\left(1-T / T_{c}\right)^{-1 / 2}$ near the critical temperature $T_{c}$. We also obtain the magnetic penetration depth $\lambda \propto\left(T_{c}-T\right)^{1 / 2}$ by adding a small external homogeneous magnetic field. The results agree with the Ginzburg-Landau theory.
\end{abstract}

PACS numbers: $11.25 . \mathrm{Tq}, 74.20 .-\mathrm{z}$

\section{INTRODUCTION}

The AdS/CFT correspondence [1, 4 , has played an important role in understanding strongly coupled gauge theories. Recently, it also has been applied to superconductivity. The key point of the holographic theories for superconductors is that in the gravity theory, a black hole coupled with matter fields will have symmetry breaking solutions. There are mainly two holographic models of superconductors with different matter sectors. The first one is an Abelian-Higgs model which is the gravity dual of an $s$-wave superconductor with a scalar order parameter. The properties of this holographic superconductor model have been studied by many authors [5-31]. The other one is an Einstein-Yang-Mills (EYM) theory in which the condensate carries angular momenta [32-43].

Gubser [44] firstly presented an argument that by coupling the Abelian Higgs model to gravity with a negative cosmological constant, one can get solutions which spontaneously break the Abelian gauge symmetry via a charged complex scalar condensate near the horizon of the black hole. Hartnoll et al [5] explored this Abelian-Higgs model of superconductivity further. They built an $s$-wave holographic (in the sense of AdS/CFT duality) superconductor with scalar order parameter which exhibits the basic features of a superconductor such as the existing of a critical temperature below which a charged condensate forms. The behaviors of the $s$-wave holographic superconductor under magnetic field have been studied in many papers [6 9, 16 19]. Especially, Maeda and Okamura [8] studied the superconducting coherence length $\xi$ of the $s$-wave holographic superconductor near the critical temperature $T_{c}$. They obtained that $\xi$ is proportional to $\left(1-T / T_{c}\right)^{-1 / 2}$, which is in agreement with the Ginzburg-Landau theory.

The other holographic superconductor model which is an EYM model with fewer parameters whose Lagrangian is determined by symmetry principles is constructed later by Gubser [45] and is shown to have spontaneous symmetry breaking solutions due to a condensate of non-Abelian gauge fields in the theory. Gubser and Pufu studied this model with both $p$-wave backgrounds and $(p+i p)$-wave backgrounds [32]. Roberts and Hartnoll studied the $(p+i p)$-wave backgrounds and found two major nonconventional features for this holographic superconductor that are different from the $s$-wave counterpart. One is the existence of a pseudogap at zero temperature, and the other is the spontaneous breaking of time reversal symmetry [33]. In our recent paper [39], we studied the phase transition properties of this model in constant external magnetic field. We found that the added magnetic field indeed suppresses the superconductivity. In the present paper, we further study the $p$-wave holographic superconductor composed of a non-Abelian gauge fields (the matter sector) and a black hole background (the gravity sector) by using perturbation theory near the critical temperature, following closely Maeda and Okamura [8]. According to the Ginzburg-Landau 
theory, the superconducting length, or the correlation length of the order parameter is an important characteristic parameter for a superconductor. Since the order parameter of the $p$-wave holographic superconductor is the vector operator dual to the charged non-Abelian gauge field, we investigate the static fluctuation of the condensed non-Abelian gauge field with nonvanishing spatial momentum along one spatial direction of the AdS boundary to get the correlation length $\xi$. A homogenous magnetic field in the field theory is added by placing a small vector potential in the matter sector. The magnetic penetration length $\lambda$ is obtained by calculating the London current of the holographic superconductor.

The organization of this paper is as follows. In Section II, we reconstruct the superconducting solution of the EYM theory which is dual to a $p$-wave superconductor by perturbation techniques. Section III is devoted to the derivation of $\xi$ by solving the eigenvalue equations from the perturbation. In Section IV we find that the London current can be induced by a homogeneous magnetic field, and the magnetic penetration length is also studied. The conclusion and some discussions are given in Section V.

\section{MODEL OF A $p$-WAVE HOLOGRAPHIC SUPERCONDUCTOR}

In this section, we review the gravity dual theory of the $p$-wave superconductor. The starting point of studying holographic superconductor at finite temperature $T$ is choosing a black hole solution with a negative cosmological constant such that the Hawking temperature of the black hole is $T$. The full EYM theory in 3+1 dimensional spacetime considered in Refs. [32, 33, 45] has the following action

$$
S_{\mathrm{EYM}}=\int \sqrt{-g} d^{4} x\left[\frac{1}{2 \kappa_{4}^{2}}\left(R+\frac{6}{L^{2}}\right)-\frac{L^{2}}{2 g_{\mathrm{YM}}^{2}} \operatorname{Tr}\left(F_{\mu \nu} F^{\mu \nu}\right)\right],
$$

where $g_{\mathrm{YM}}$ is the gauge coupling constant and $F_{\mu \nu}=T^{a} F_{\mu \nu}^{a}=\partial_{\mu} A_{\nu}-\partial_{\nu} A_{\mu}-i\left[A_{\mu}, A_{\nu}\right]$ is the field strength of the gauge field $A=A_{\mu} d x^{\mu}=T^{a} A_{\mu}^{a} d x^{\mu}$. For the $S U(2)$ gauge symmetry, $\left[T^{a}, T^{b}\right]=$ $i \epsilon^{a b c} T^{c}$ and $\operatorname{Tr}\left(T^{a} T^{b}\right)=\delta^{a b} / 2$, where $\epsilon^{a b c}$ is the totally antisymmetric tensor with $\epsilon^{123}=1$. The Yang-Mills Lagrangian becomes $\operatorname{Tr}\left(F_{\mu \nu} F^{\mu \nu}\right)=F_{\mu \nu}^{a} F^{a \mu \nu} / 2$ with the field strength components $F_{\mu \nu}^{a}=$ $\partial_{\mu} A_{\nu}^{a}-\partial_{\nu} A_{\mu}^{a}+\epsilon^{a b c} A_{\mu}^{b} A_{\nu}^{c}$.

Working in the probe limit in which the matter fields do not backreact on the metric as in Refs. [32, 33, 45] and taking the planar Schwarzchild-AdS ansatz, the black hole metric reads (we use mostly plus signature for the metric)

$$
d s^{2}=-f(r) d t^{2}+\frac{d r^{2}}{f(r)}+\frac{r^{2}}{L^{2}}\left(d x^{2}+d y^{2}\right),
$$

where the metric function $f(r)$ is

$$
f(r)=\frac{r^{2}}{L^{2}}\left(1-\frac{r_{0}^{3}}{r^{3}}\right) .
$$

$L$ and $r_{0}$ are the radius of the AdS spacetime and the horizon radius of the black hole, respectively. They determine the Hawking temperature of the black hole,

$$
T=\frac{3 r_{0}}{4 \pi L^{2}},
$$

which is also the temperature of the dual gauge theory living on the boundary of the AdS spacetime. Now we introduce a new coordinate $z=r_{0} / r$. The metric (II.2) then becomes

$$
d s^{2}=\frac{L^{2} \beta^{2}(T)}{z^{2}}\left(-h(z) d t^{2}+d x^{2}+d y^{2}\right)+\frac{L^{2} d z^{2}}{z^{2} h(z)},
$$


where $h(z)=1-z^{3}$ and $\beta(T)=r_{0} / L^{2}=4 \pi T / 3$.

Using the Euler-Lagrange equations, one can obtain the equations of motion for the gauge fields,

$$
\frac{1}{\sqrt{-g}} \partial_{\mu}\left(\sqrt{-g} F^{a \mu \nu}\right)+\epsilon^{a b c} A_{u}^{b} F^{c \mu \nu}=0 .
$$

For the $p$-wave backgrounds, the ansatz [32] takes the following form,

$$
A=\phi(z) T^{3} d t+w(z) T^{1} d x .
$$

With this ansatz, we can derive the equations of motion for the two dimensionless quantities $\tilde{w}(z)=$ $w(z) / \beta(T)$ and $\tilde{\phi}(z)=\phi(z) / \beta(T)$,

$$
\frac{d}{d z}\left(h(z) \frac{d \tilde{w}}{d z}\right)+\frac{\tilde{\phi}^{2} \tilde{w}}{h(z)}=0,
$$

and

$$
\frac{d^{2} \tilde{\phi}}{d z^{2}}-\frac{\tilde{\phi} \tilde{w}^{2}}{h(z)}=0
$$

Here the $U(1)$ subgroup of $S U(2)$ generated by $T^{3}$ is identified to the electromagnetic gauge group [45] and $\phi$ is the electrostatic potential, which must vanish at the horizon for the gauge field $A$ to be well-defined, but need not vanish at infinity. Thus the black hole can carry charge through the condensate $w$, which spontaneously breaks the $U(1)$ gauge symmetry. This is a Higgs mechanism, but there are Goldstone bosons corresponding to changing the directions of the condensate in real space or gauge space. They must be visible in the bulk as normal modes or (more likely) quasi-normal modes.

The exact solution of the equations of motion is

$$
\tilde{w}=0, \tilde{\phi}=\mu / \beta(T)-q z=q(1-z),
$$

where $\mu$ is interpreted as the chemical potential of the field theory. This trivial solution is parameterized by the dimensionless constant $q$, which is related to the charge density of the dual field theory which couples to $\mu$ as

$$
\left\langle J^{0}\right\rangle=\frac{\delta S_{\text {on-shell boundary }}}{\delta A_{0}^{3}}=\frac{1}{2 g_{\mathrm{YM}}^{2}} \beta^{2}(T) q .
$$

The superconducting solution with non-vanishing $\tilde{w}$ takes the following asymptotic form at the AdS boundary,

$$
\begin{gathered}
\tilde{w}=\frac{\langle\mathcal{O}\rangle}{\sqrt{2}} z+\cdots, \\
\tilde{\phi}=\mu / \beta(T)-q z+\cdots,
\end{gathered}
$$

where $\langle\mathcal{O}\rangle$ is the condensate of the charged operator dual to the field $w$ and is the order parameter for the superconductivity phase. We demand that the constant term vanish since we require that there is no source term in field theory action for the operator $\langle\mathcal{O}\rangle[32,33]$.

According to numerical calculations [32], the order parameter behaves as

$$
\langle\mathcal{O}\rangle \sim\left(1-T / T_{c}\right)^{1 / 2}
$$


near the critical phase transition point. For the reason of continuity, the solution at the critical temperature should be

$$
\tilde{w}_{c}=0, \tilde{\phi}_{c}=q_{c}(1-z) .
$$

The non-trivial solution near the critical temperature can be obtained by a perturbation expansion in terms of $\epsilon=\left(1-T / T_{c}\right)$ since $\epsilon$ is a small parameter. We expand $w(z)$ and $\phi \tilde{(z)}$ as

$$
\begin{gathered}
\tilde{w}(z)=\epsilon^{1 / 2} \tilde{w}_{1}(z)+\epsilon^{3 / 2} \tilde{w}_{2}(z)+\cdots, \\
\tilde{\phi}(z)=\tilde{\phi}_{c}(z)+\epsilon \tilde{\phi}_{1}(z)+\cdots .
\end{gathered}
$$

Substituting Eq.(II.16) and Eq.(II.17) into Eq.(II.9) and Eq.(II.8), we obtain equations for $\tilde{w}_{1}$ and $\tilde{\phi}_{1}$,

$$
\begin{gathered}
\mathcal{L}_{w} \tilde{w}_{1}(z)=0, \\
\frac{d^{2} \tilde{\phi}_{1}(z)}{d z^{2}}-\frac{\tilde{\phi}_{c}(z) \tilde{w}_{1}^{2}(z)}{h(z)}=0,
\end{gathered}
$$

where we have defined the following operator,

$$
\mathcal{L}_{w}=-\left(\frac{d}{d z} h(z) \frac{d}{d z}+\frac{\tilde{\phi}_{c}^{2}(z)}{h(z)}\right) .
$$

\section{THE SUPERCONDUCTING COHERENCE LENGTH}

As an important parameter for superconductor, the superconducting coherence length is obtained from the complex pole of the static correlation function of the order parameter in Fourier space:

$$
\langle\tilde{\mathcal{O}}(\vec{k}) \tilde{\mathcal{O}}(-\vec{k})\rangle \sim \frac{1}{|\vec{k}|^{2}+1 / \xi^{2}} .
$$

The pole $|\vec{k}|^{2}$ can be calculated in the probe limit by perturbing the fields $(\tilde{w}, \tilde{\phi})$ in the equations of motion Eq.(1I.9) and Eq.(1I.8). It is enough to consider a linear perturbation with fluctuation of the field in the $y$-direction which takes the following form,

$$
\begin{aligned}
\delta \tilde{\phi}(z, y) d t & =[\Phi(z, k) d t] e^{i k y}, \\
\delta \tilde{w}(z, y) & =[W(z, k)] e^{i k y} .
\end{aligned}
$$

Using this perturbation, we get the following linearized equations for $W$ and $\Phi$ :

$$
\begin{gathered}
\tilde{k}^{2} W=\left(\frac{d}{d z} h(z) \frac{d}{d z}+\frac{\tilde{\phi}^{2}}{h(z)}\right) W+\frac{2 \tilde{w} \tilde{\phi}}{h(z)} \Phi, \\
\tilde{k}^{2} \Phi=\left(h(z) \frac{d^{2}}{d z^{2}}-\tilde{w}^{2}\right) \Phi-2 \tilde{\phi} \tilde{w} W
\end{gathered}
$$


where $\tilde{k}=k / \beta(T)$ is dimensionless.

Now, our task is to solve the eigenvalue equations near $T_{c}$ analytically. Using the perturbation expansions in Eq.(II.16) and Eq.(II.17), we get

$$
\begin{aligned}
& -\tilde{k}^{2} W=\left(\mathcal{L}_{w}-\frac{2 \epsilon \tilde{\phi}_{c} \tilde{w}_{1}}{h(z)}\right) W-\frac{2 \epsilon^{1 / 2} \tilde{\phi}_{c} \tilde{w}_{1}}{h(z)} \Phi, \\
& -\tilde{k}^{2} \Phi=\left(-h(z) \frac{d^{2}}{d z^{2}}+\epsilon \tilde{w}_{1}^{2}\right) \Phi+2 \epsilon^{1 / 2} \tilde{\phi}_{c} \tilde{w}_{1} W .
\end{aligned}
$$

The boundary conditions for the two equations are

$$
W(1)=\text { regular }, \quad \Phi(1)=0
$$

at the horizon and

$$
\begin{gathered}
W(z)=(\text { const }) \times z+O\left(z^{2}\right), \\
\Phi(z)=(\text { const }) \times z+O\left(z^{2}\right)
\end{gathered}
$$

near the AdS boundary $z=0$.

The trivial solution is the zeroth order solution $\Phi_{0}$ and $W_{0}$ with $\tilde{k}=0$,

$$
\Phi=0, \quad W_{0}=\tilde{w}_{1},
$$

where equation (II.18) is used. The non-trivial solutions can be obtained by a series expansion around the zeroth order solution in $\epsilon$,

$$
\begin{gathered}
W=\tilde{w}_{1}+\epsilon W_{1}+\epsilon^{2} W_{2}+\cdots, \\
\Phi=\epsilon^{1 / 2} \Phi_{1}+\epsilon^{3 / 2} \Phi_{2}+\cdots \\
\tilde{k}^{2}=\epsilon \tilde{k}_{1}^{2}+\epsilon^{2} \tilde{k}_{2}^{2}+\cdots
\end{gathered}
$$

Using this expansion in Eq.(III.6) and Eq.(III.7), one has

$$
\begin{gathered}
-\tilde{k}_{1}^{2} \tilde{w}_{1}=\mathcal{L}_{w} W_{1}-\frac{2 \tilde{\phi}_{c} \tilde{w}_{1}}{h(z)}\left(\tilde{\phi}_{1}+\Phi_{1}\right), \\
\frac{d^{2} \Phi_{1}}{d z^{2}}=\frac{2 \tilde{\phi}_{c} \tilde{w}_{1}^{2}}{h(z)}=\frac{2 d^{2} \tilde{\phi}_{1}}{d z^{2}} .
\end{gathered}
$$

Eq. (III.15) can be solved for $\tilde{k}$ by defining an inner product for the states $w_{I}$ and $w_{I I}$,

$$
\left\langle w_{I} \mid w_{I I}\right\rangle=\int_{0}^{1} d z w_{I}^{*}(z) w_{I I}(z) .
$$

Using this inner product for Eq. (III.15) and $\tilde{w}_{1}$, with the fact that $\mathcal{L}_{w} \tilde{w}_{1}=0$, we have

$$
\tilde{k}_{1}^{2}\left\langle\tilde{w}_{1} \mid \tilde{w}_{1}\right\rangle=\left\langle\tilde{w}_{1} \mid \frac{2 \tilde{\phi}_{c} \tilde{w}_{1}}{h(z)} \tilde{\phi}_{1}\right\rangle+2 \int_{0}^{1} d z \frac{\tilde{\phi}_{c} \tilde{w}_{1}^{2}}{h(z)} \Phi_{1} .
$$


The first term of the above equation vanishes, which can be seen from the Hermiticity of $\mathcal{L}_{w}$ and

$$
\mathcal{L}_{w} \tilde{w}_{2}=\frac{2 \tilde{\phi}_{c} \tilde{w}_{1}}{h(z)} \tilde{\phi}_{1}
$$

Eq. (III.19) is the equation of motion for $\tilde{w}_{2}$ defined in (II.16). Using the fact that $\tilde{k}^{2}=\epsilon \tilde{k}_{1}^{2}$, the eigenvalue $\tilde{k}$ in a first order approximation can be written as

$$
\tilde{k}^{2}=\epsilon \frac{N}{D}+O\left(\epsilon^{2}\right)
$$

where

$$
N=2 \int_{0}^{1} d z \frac{\tilde{\phi}_{c} \tilde{w}_{1}^{2}}{h(z)} \Phi_{1} \quad \text { and } \quad D=\int_{0}^{1} d z \psi_{1}^{2} .
$$

Finally, the superconducting coherence length is given by

$$
\xi=\frac{\epsilon^{-1 / 2}}{\beta\left(T_{c}\right)} \sqrt{\frac{D}{N}}+O\left(\epsilon^{2}\right) \propto\left(1-\frac{T}{T_{c}}\right)^{-1 / 2} .
$$

We have thus obtained the same critical exponent $(-1 / 2)$ for $\xi$ as given by the Ginzburg-Landau theory.

\section{THE LONDON EQUATION AND MAGNETIC PENETRATION LENGTH}

In order to calculate the magnetic penetration length for the holographic superconductor, we add a homogenous external magnetic field by assuming a perturbative non-zero $\delta A_{y}^{3}(z, x)=b(z) x$, where $\lim _{z \rightarrow 0} \delta A_{y}^{3}(z, x)=B x$. Then the magnetic field in the field theory is $F_{x y}=\partial_{x} \delta A_{y}=B[8]$. We still work in the probe limit where the magnetic field does not affect the metric. If we only focus on the neighborhood of $x=0$, the equation of motion for $b(z)$ can be treated as decoupled from $\tilde{w}$,

$$
\left(\frac{d}{d z} h(z) \frac{d}{d z}-\tilde{w}^{2}\right) b(z)=0,
$$

where $b(z)$ must satisfie the regularity boundary condition at the horizon $z=1$. This equation can also be solved by perturbation. We can expand $b(z)$ as

$$
b(z)=b_{0}(z)+\epsilon b_{1}(z)+\cdots .
$$

Substituting this expansion and Eq.(II.16) into Eq.(IV.1), we obtain the equations,

$$
\begin{gathered}
\frac{d}{d z} h(z) \frac{d}{d z} b_{0}(z)=0, \\
\frac{d}{d z} h(z) \frac{d}{d z} b_{1}(z)-\tilde{w}_{1}^{2}(z) b_{0}(z)=0 .
\end{gathered}
$$

The solution of Eq. (IV.3), which satisfies the required boundary conditions is

$$
b_{0}(z)=C,
$$


where $C=B$ is a constant since the condition $\lim _{z \rightarrow 0} b(z)=B$ must be satisfied. So the solution of Eq.(IV.4) should be,

$$
\frac{d b_{1}}{d z}=-\frac{B}{h(z)} \int_{z}^{1} d z_{0} \tilde{w}_{1}^{2}\left(z_{0}\right)
$$

Integrating the above equation. we have,

$$
b(z)=B-\epsilon B \int_{0}^{z} \frac{d z_{1}}{h\left(z_{1}\right)} \int_{z_{1}}^{1} d z_{0} \tilde{w}_{1}^{2}\left(z_{0}\right)+O\left(\epsilon^{2}\right) .
$$

Using the fact that $B=\lim _{z \rightarrow 0} b(z)$ and $\delta A_{y}^{3(0)}(x)=\lim _{z \rightarrow 0} \delta A_{y}^{3}(z, x)$, we can rewrite Eq. (IV.7) as

$$
\delta A_{y}^{3}(z, x)=\delta A_{z}^{3(0)}(x)\left(1-\epsilon \int_{0}^{z} \frac{d z_{1}}{h\left(z_{1}\right)} \int_{z_{1}}^{1} d z_{0} \tilde{w}_{1}^{2}\left(z_{0}\right)\right)+O\left(\epsilon^{2}\right) .
$$

According to the AdS/CFT dictionary, we can read out the current $\left\langle J_{y}(x)\right\rangle$ near $T_{c}$ to be,

$$
\left\langle J_{y}(x)\right\rangle=-\frac{L^{2}}{g_{Y M}^{2}}\left(\frac{4 \pi T_{c}}{3}\right)\left(1-\frac{T}{T_{c}}\right) \int_{0}^{1} d z \tilde{w}_{1}^{2}(z) \delta A_{y}^{3(0)}(x)+O\left(\epsilon^{2}\right),
$$

or

$$
\left\langle J_{y}(x)\right\rangle \sim-T_{c} \epsilon \delta A_{y}^{3(0)}(x) .
$$

This is similar to the London equation for real world superconductors,

$$
\boldsymbol{J}=-\frac{e_{*}^{2}}{m_{*}} \psi^{2} \boldsymbol{A}=-e_{*} n_{s} \boldsymbol{A},
$$

where $e_{*}$ and $m_{*}$ are effective charge and mass of the order parameter respectively, and $n_{s}$ is the superfluid number density.

Comparing Eq.(IV.10) and Eq.(IV.11), we find that the superfluid density $n_{s}$ near the critical point in the field theory is

$$
n_{s} \sim \epsilon T_{c} \sim T_{c}-T .
$$

According to the Ginzburg-Landau theory, the magnetic penetration depth $\lambda$ is given by

$$
\lambda \sim 1 / \sqrt{n_{s}} .
$$

Then, we get the behavior of $\lambda$ in the vicinity of the critical temperature,

$$
\lambda \propto\left(T_{c}-T\right)^{-1 / 2},
$$

which is the expected result as in the Ginzburg-Landau theory.

\section{CONCLUSION AND DISCUSSIONS}

For the EYM theory with a $p$-wave backgrounds, we investigated the linear fluctuation of the condensation solution under the probe limit. By solving the linearized eigenvalue equations with only spatial momentum along one spatial direction by the perturbation method, we obtain that the 
correlation length $\xi$ diverges as $\xi \sim\left(1-T_{c} / T\right)^{-1 / 2}$ at the critical temperature. We also find that the magnetic penetration length behaves as $\lambda \sim\left(T_{c}-T\right)^{-1 / 2}$ near the critical temperature. The London type equation Eq. (IV.10) implies a Meissner effect in the superconductor. These results are consistent with the Ginzburg-Landau theory, which supports the idea that the non-Abelian holographic model can be used to describe superconductors. Our results are similar to those of the $s$-wave holographic superconductor studied by Maeda and Okamura [8]. Though the holographic models have made many achievements, it is still a unsolved question why the Ginzburg-Landau behavior is expected and when one would expect deviations from it in these models. Recently, vortex solutions of the $s$-wave holographic superconductor in homogeneous external magnetic field have been studied in Ref. [16-19]. It will be interesting to study possible localized vortex solutions in the $p$-wave holographic superconductor.

\section{ACKNOWLEDGEMENT}

We thank professor C. P. Herzog and professor S. S. Gubser for help. We also thank Wei-Ming Sun for helpful discussions. This work is supported in part by the National Natural Science Foundation of China (under Grant No. 10775069) and the Research Fund for the Doctoral Program of Higher Education (Grant Nos. 20060284020 and 20080284020).

[1] J. M. Maldacena, "The large N limit of superconformal field theories and supergravity," Adv. Theor. Math. Phys. 2 (1998) 231 arXiv:hep-th/9711200.

[2] S. S. Gubser, I. R. Klebanov and A. M. Polyakov, "Gauge Theory Correlators from Non-Critical String Theory," Phys. Lett. B 428, 105 (1998) arXiv:hep-th/9802109.

[3] E. Witten, "Anti-de Sitter space and holography," Adv. Theor. Math. Phys. 2, 253 (1998) arXiv:hep-th/9802150.

[4] O. Aharony, S. S. Gubser, J. M. Maldacena, H. Ooguri, and Y. Oz, "Large N Field Theories, String Theory and Gravity," Phys. Rept. 323, 183 (2000) arXiv:hep-th/9905111.

[5] S. A. Hartnoll, C. P. Herzog and G. T. Horowitz, "Building a Holographic Superconductor," Phys. Rev. Lett. 101, 031601 (2008) arXiv:0803.3295 [hep-th]].

[6] E. Nakano and W. Y. Wen, "Critical Magnetic Field In A Holographic Superconductor," Phys. Rev. D 78, 046004 (2008) arXiv:0804.3180 [hep-th]].

[7] T. Albash and C. V. Johnson, "A Holographic Superconductor in an External Magnetic Field," JHEP 0809, 121 (2008) arXiv:0804.3466 [hep-th]].

[8] K. Maeda and T. Okamura, "Characteristic length of an AdS/CFT superconductor," Phys. Rev. D 78, 106006 (2008) arXiv:0809.3079 [hep-th]].

[9] S. A. Hartnoll, C. P. Herzog and G. T. Horowitz, "Holographic Superconductors," JHEP 0812, 015 (2008) arXiv:0810.1563 [hep-th]].

[10] C. P. Herzog, P. K. Kovtun and D. T. Son, "Holographic model of superfluidity," Phys. Rev. D 79, 066002 (2009) arXiv:0809.4870 [hep-th]].

[11] G.T. Horowitz and M.M. Roberts, "Holographic Superconductors with Various Condensates,"Phys. Rev. D 78126008 (2008) arXiv:0810.1077 [hep-th]].

[12] S. Franco, A. Garcia-Garcia, D. Rodriguez-Gomez ,"A general class of holographic superconductors," arXiv:0906.1214 [hep-th]].

[13] I. Amado, M. Kaminski, K. Landsteiner, "Hydrodynamics of Holographic Superconductors," JHEP 0905, 021 (2009).

[14] K. Maeda, M. Natsuume, T. Okamura, "Universality class of holographic superconductors," Phys. Rev. D 79, 126004 (2009).

[15] Youngman Kim, Yumi Ko, Sang-Jin Sin, “ Density driven symmetry breaking and Butterfly effect in holographic superconductors ," arXiv:0904.4567 [hep-th]]. 
[16] T. Albash, C. V. Johnson, "Phases of Holographic Superconductors in an External Magnetic," arXiv:0906.0519 [hep-th]].

[17] T. Albash, C. V. Johnson, "Vortex and Droplet Engineering in Holographic Superconductors," arXiv:0906.1795 [hep-th]].

[18] M. Montull, A. Pomarol, P. J. Silva, "The Holographic Superconductor Vortex," Phys. Rev. Lett 103 091601 (2009) arXiv:0906.2396 [hep-th]].

[19] K. Maeda, M. Natsuume, T. Okamura," "Vortex lattice for a holographic superconductor," arXiv:0910.4475 [hep-th]].

[20] V. Keranen, E. Keski-Vakkuri, S. Nowling, K. P. Yogendran, "Inhomogeneous Structures in Holographic Superfluids: II. Vortices," arXiv:0912.4280 [hep-th]].

[21] T. Nishioka, S. Ryu, T. Takayanagi, "Holographic Superconductor/Insulator Transition at Zero Temperature," arXiv:0911.0962 [hep-th]].

[22] A. Konoplya, A. Zhidenko, "Holographic conductivity of zero temperature superconductors," arXiv:0909.2138 [hep-th]].

[23] G. T. Horowitz, M. M. Roberts, "Zero Temperature Limit of Holographic Superconductors," JHEP 11 (2009) 015 arXiv:0908.3677[ [hep-th]].

[24] S. S. Gubser, A. Nellore, "Ground states of holographic superconductors," arXiv:0908.1972 [hep-th]].

[25] S. J. Sin, S. S. Xu, Y. Zhou, "Holographic Superconductor for a Lifshitz fixed point," arXiv:0909.4857 [hep-th]].

[26] E.J.Brynjolfsson, U.H. Danielsson, L. Thorlacius, T. Zingg, " Holographic Superconductors with Lifshitz Scaling," arXiv:0908.2611 [hep-th]].

[27] O. C. Umeh, "Scanning the Parameter Space of Holographic Superconductors," JHEP 0908: 062,2009 arXiv:0907.3136 [hep-th]].

[28] C. P. Herzog, A. Yarom, "Sound modes in holographic superfluids," arXiv:0906.4810 [hep-th]].

[29] V. Keranen, E. Keski-Vakkuri, S. Nowling, K. P. Yogendran, "Dark Solitons in Holographic Superfluids," arXiv:0906.5217 v3 [hep-th]].

[30] V. Keranen, E. Keski-Vakkuri, S. Nowling, K. P. Yogendran, "Inhomogeneous Structures in Holographic Superfluids: I. Dark Solitons," arXiv:0911.1866 [hep-th]].

[31] J. W. Chen, Y. J. Kao and W. Y. Wen, "Peak-Dip-Hump from Holographic Superconductivity," arXiv:0911.2821 [hep-th]].

[32] S. S. Gubser and S. S. Pufu, "The gravity dual of a p-wave superconductor," JHEP 0811, 033 (2008) arXiv:0805.2960 [hep-th]].

[33] M. M. Roberts and S. A. Hartnoll, "Pseudogap and time reversal breaking in a holographic superconductor," JHEP 0808, 035 (2008) arXiv:0805.3898 [hep-th]].

[34] M. Ammon, J. Erdmenger, M. Kaminski and P. Kerner, "Superconductivity from gauge/gravity duality with flavor," Phys. Lett. B680 (2009) arXiv:0810.2316 [hep-th]].

[35] M. Ammon, J. Erdmenger, M. Kaminski and P. Kerner, "Flavor Superconductivity from Gauge/Gravity Duality," JHEP 10 (2009) 067 arXiv:0903.1864 [hep-th]].

[36] P. Basu, J. He, A. Mukherjee and H. H. Shieh, "Superconductivity from D3/D7: Holographic Pion Superfluid," arXiv:0810.3970 [hep-th]].

[37] C. P. Herzog and S. S. Pufu, "The Second Sound of SU(2)," JHEP 04 (2009) 126 arXiv:0902.0409 [hep-th]].

[38] J. Sonner, "A Rotating Holographic Superconductor," Phys. Rev. D 80, 084031 arXiv:0903.0627 [hepth]].

[39] H. Zeng, Z. Fan, Z. Ren, "Time Reversal Symmetry Breaking Holographic Superconductor in Constant External Magnetic Field,"Phys. Rev. D 80, 066001 arXiv:0903.2323 [hep-th]].

[40] K. Peeters, J. Powell, M. Zamaklar, "Exploring colourful holographic superconductors," JHEP 0909, 101, 2009 arXiv:0907.1508 [hep-th]].

[41] C. P. Herzog, "Lectures on Holographic Superfluidity and Superconductivity," J. Phys. A 42 (2009) 343001 arXiv:0904.1975 [hep-th]].

[42] Pallab. Basu, Jianyang. He, Anindya. Mukherjee, Hsien-Hang. Shieh, "Hard-gapped Holographic Superconductors," arXiv:0911.4999.

[43] Martin Ammon, Johanna Erdmenger, Viviane Grass, Patrick Kerner, Andy O’Bannon, " On Holographic p-wave Superfluids with Back-reaction," arXiv:0912.3515.

[44] S. S. Gubser, "Breaking an Abelian gauge symmetry near a black hole horizon," Phys. Rev. D 78, 065034 (2008) [arXiv:0801.2977 [hep-th]]. 
[45] S. S. Gubser, "Colorful horizons with charge in anti-de Sitter space," Phys. Rev. Lett. 101, 191601 (2008) arXiv:0803.3483 [hep-th]]. 